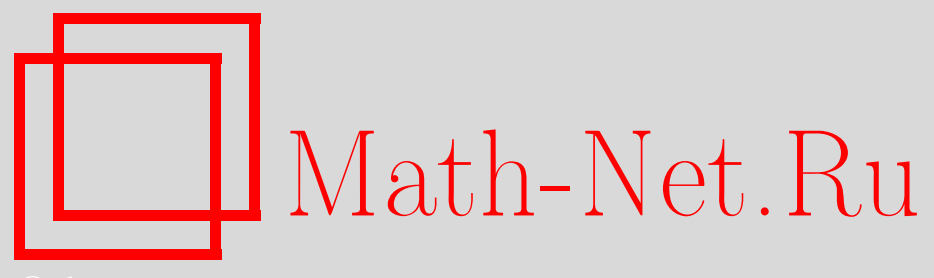

С. Ю. Доброхотов, Б. Тироцци, А. И. Шафаревич, Представления быстроубывающих функций каноническим оператором Маслова, Матем. заметки, 2007, том 82, выпуск 5, 792-796

DOI: https://doi.org/10.4213/mzm4090

Использование Общероссийского математического портала Math-Net.Ru подразумевает, что вы прочитали и согласны с пользовательским соглашением http: //www . mathnet.ru/rus/agreement

Параметры загрузки:

IP : 54.224 .135 .184

26 апреля 2023 г., 11:05:29






\section{Представления быстроубывающих функций каноническим оператором Маслова}

\section{С. Ю. Доброхотов, Б. Тироцци, А. И. Шафаревич}

1. Основной результат. Пусть $\mu$ - малый положительный параметр, а $V(y), y \in$ $\mathbb{R}^{2}$, - гладкая функция, убывающая на бесконечности как $1 /|y|^{k}, k \geqslant 2$, причем производные $\partial V / \partial y_{j}$ убывают как $1 /|y|^{k+1}$. Функция $V(x / \mu)$ локализована в окрестности нуля. Обозначим через $\widetilde{V}(p)$ ее преобразование Фурье:

$$
\widetilde{V}(p)=\frac{1}{(2 \pi)^{n / 2}} \int_{\mathbb{R}_{y}^{n}} e^{-i\langle p, y\rangle} V(y) d y .
$$

Здесь $\langle\cdot, \cdot\rangle$ - вещественное скалярное произведение. Тогда справедлива формула

$$
V\left(\frac{x}{\mu}\right)=\frac{1}{(2 \pi)^{n / 2}} \int_{\mathbb{R}_{p}^{n}} e^{(i / \mu)\langle p, x\rangle} \widetilde{V}(p) d p .
$$

Функция $V(x / \mu)$ быстро убывает при $\mu \rightarrow+0$ вне малой окрестности точки $x=0$. Имея в виду изучение асимптотических решений задачи Коши с начальными данными вида (2) для уравнений в частных производных выражение в правой части можно записать в виде канонического оператора Маслова [1] $K_{\Lambda_{\delta}}^{\mu}$ на лагранжевом многообразии (плоскости) $\Lambda_{\delta}=\left\{p=\alpha, x=0, \alpha \in \mathbb{R}^{n}\right\}$, действующим на функцию $\widetilde{V}(\alpha)\left(\right.$ на $\left.\Lambda_{\delta}\right)$ :

$$
V\left(\frac{x}{\mu}\right)=\left(\frac{\mu}{i}\right)^{n / 2} K_{\Lambda_{\delta}}^{\mu}[\widetilde{V}(\alpha)] .
$$

Мы полагаем здесь и всюду ниже $\sqrt{i}=e^{i \pi / 4}$. Последнее равенство легко следует из определения канонического оператора с учетом того, что

(a) на $\Lambda^{2}$ действие $\int\langle p, d x\rangle=0$;

(b) все точки на $\Lambda_{\delta}$ фокальные и бесконечно вырождены, так что $\Lambda_{\delta}$ покрывается одной фокальной картой $x=0$ с координатами $\left(p_{1}, \ldots, p_{n}\right)$;

(c) якобиан $\operatorname{det} \partial p /\left.\partial \alpha\right|_{\Lambda_{\delta}} \equiv 1$.

Функция $V(x / \mu) / \mu^{n}$ определяет некоторую $\delta$-образную последовательность: при $\mu \rightarrow$ +0 функция $V(x / \mu) / \mu^{n} \rightarrow \delta(x) \int_{\mathbb{R}^{n}} V(y) d y$, где $\delta(x)$ - функции Дирака, и правая часть в (3) есть интегральное представление для $\delta$-образной последовательности. По этой причине лагранжево многообразие $\Lambda_{\delta}$ в представлении (3) такое же, как в формуле для одного из представлений $\delta$-функции через канонический оператор Маслова [1]. Такое представление хорошо работает при изучении локализованных решений в случае, когда символ (гамильтониан) $H$ изучаемого дифференциального уравнения - гладкая функция, например, в случае уравнения Шрёдингера: тогда $H=p^{2} / 2+v(x)$. В случае, когда гамильтониан $H$ - негладкая функция (например, для волнового уравнения $H=|p| c(x)$ ), представление (3) оказывается не очень эффективным с точки зрения получения простых асимптотических формул [2], [3].

В этой заметке мы даем новое представление функции $V(x / \mu)$ с помощью канонического оператора Маслова в виде, отличном от (3) и существенно более эффективном при

Работа выполнена при поддержке Российского фонда фундаментальных исследований (грант № 05-01-00968) и соглашения о сотрудничестве между ИПМех PAH и Университетом "La Sapienza".

(С) С. Ю. Доврохотов, Б. Тироцци, А. И. ШАФАРевич, 2007 
изучении решений задачи Коши с локализованными начальными данными для (систем) гиперболических уравнений [4]. Наш центральный результат состоит в наблюдении, согласно которому функция $V(x / \mu)$ может быть представлена в виде интеграла по вспомогательной переменной $\rho$ от канонического оператора Маслова с "квазиклассическим" параметром $h=\mu / \rho$, определенном на лагранжевом многообразии, отличном от $\Lambda_{\delta}$ и действующим на функцию $f$, связанную с $\widetilde{V}$. Ограничиваясь для большей наглядности случаем $n=2$, мы покажем, что

$$
V\left(\frac{x}{\mu}\right)=\sqrt{\frac{\mu C_{0}}{2 \pi}} e^{-i \pi / 4} \int_{0}^{\infty} K_{\Lambda_{0}^{2}}^{\mu / \rho} \sqrt{\rho} \widetilde{V}(\rho \mathbf{n}(\psi)) d \rho+O(\mu) .
$$

Здесь

$$
\mathbf{n}(\psi)=\left(\begin{array}{c}
\cos (\psi) \\
\sin (\psi)
\end{array}\right)
$$

лагранжево многообразие

$$
\Lambda_{0}^{2}=\left\{p=\mathbf{n}(\psi), x=C_{0} \mathbf{n}(\psi) \alpha\right\}
$$

$C_{0}$ - произвольная положительная константа, $\alpha \in \mathbb{R}, \psi \in \mathbb{S}=[0,2 \pi)$ - координаты на $\Lambda_{0}^{2}$, а $K_{\Lambda_{0}^{2}}^{h}-$ канонический оператор Маслова с формой площади $d \alpha \wedge d \psi$ и отмеченной точкой, лежащей на окружности $\alpha=0$. С точки зрения перехода $V(x / \mu)$ при $\mu \rightarrow+0$ к $\delta$-функции представление (4) соответствует переходу к полярным координатам в интеграле (2). Заметим также, что многообразие $\Lambda_{0}^{2}$ хорошо известно в теории псевдодифференциальных операторов и их применения к задачам о распространении особенностей [5].

Более полно результат, касающийся (4), мы приведем ниже в виде теоремы 1. Для доказательства (4) предварительно напомним определения объектов, относящихся к каноническому оператору.

\section{2. Лагранжево многообразие $\Lambda_{0}^{2}$, его свойства и функции на нем.}

2.1. Инвариантность. Ясно, что $\Lambda_{0}^{2}$ - двумерное лагранжево многообразие, диффеоморфное цилиндру. Отметим, что оно может быть получено сдвигом окружности $S^{1}=\{p=\mathbf{n}(\psi), x=0\}$ вдоль траекторий гамильтоновой системы с гамильтонианом $\mathscr{H}=|p| C_{0}: \Lambda_{0}^{2}=\bigcup_{-\infty<\alpha<\infty} g_{\mathscr{H}}^{\alpha} S^{1}$. Здесь, как обычно, $g_{\mathscr{H}}^{t}$ обозначает фазовый поток соответствующей гамильтоновой системы $\dot{x}=p /|p| C_{0}, \dot{p}=0,\left.p\right|_{t=0}=\mathbf{n}(\psi),\left.x\right|_{t=0}=0$. Координата $\alpha$ играет роль времени $t$, т.е. для каждой точки $(\psi, \alpha) \in \Lambda_{0}^{2} g_{\mathscr{H}}^{t}(\psi, \alpha)=(\psi, \alpha+t)$.

2.2. Цикл особенностей проекции на плоскость $p=0$ и класс Маслова. Проекция многообразия $\Lambda_{0}^{2}$ на плоскость $p=0$ устроена следующим образом. Над каждой точкой плоскости, кроме нуля, расположено две точки цилиндра $\Lambda_{0}^{2}$, причем дифференциал проекции в этих точках отличен от нуля. Над нулем висит окружность $\alpha=0$, в точках которой ранг дифференциала падает на единицу; это - цикл особенностей. Ясно, что поскольку любой цикл на $\Lambda_{0}^{2}$ можно сдвинуть так, что он не будет пересекать цикл особенностей, класс Маслова многообразия $\Lambda_{0}^{2}$ равен нулю (другими словами, индекс Маслова любой замкнутой кривой равен нулю).

2.3. Форма действия. Поскольку базисный цикл на $\Lambda_{0}^{2}$ можно выбрать лежащим в плоскости $x=0$, интеграл от формы действия $\langle p, d x\rangle$ по любой замкнутой кривой равен нулю и эта форма точна: $\langle p, d x\rangle=d\left(C_{0} \alpha\right)$. Положим $\int\langle p, d x\rangle=C_{0} \alpha$.

2.4. Канонические карты. Выберем канонические карты на $\Lambda_{0}^{2}$ следующим образом. Каждая карта гомеоморфна полосе и представляет собой декартово произведение прямой $\alpha \in \mathbb{R}$ на дугу окружности. Карта $\Omega_{1}$ соответствует дуге $-\pi / 4-\zeta<\psi<\pi / 4+\zeta$, карта $\Omega_{2}-$ дуге $\pi / 4-\zeta<\psi<3 \pi / 4+\zeta$, карта $\Omega_{3}$ - дуге $3 \pi / 4-\zeta<\psi<5 \pi / 4+\zeta$ и, наконец, карта 
$\Omega_{4}$ - дуге $5 \pi / 4-\zeta<\psi<7 \pi / 4+\zeta$. Здесь и далее $\zeta$ - достаточно малое положительное число. В качестве канонических координат в картах $\Omega_{1}, \Omega_{3}$ выберем $x_{1}, p_{2}$, а в картах $\Omega_{2}, \Omega_{4}-x_{2}, p_{1}$.

2.5. Форма площади. Форма площади в канонических координатах есть

$$
d \alpha \wedge d \psi=\frac{1}{C_{0} \sin ^{2} \psi} d p_{1} \wedge d x_{2}=\frac{1}{C_{0} \cos ^{2} \psi} d x_{1} \wedge d p_{2}=\frac{1}{C_{0} \alpha} d x_{1} \wedge d x_{2} .
$$

2.6. Разбиение единицы. Обозначим через $\mathbf{e}_{j}$ разбиение единицы, подчиненное покрытию $\Omega_{j}$; это - четыре функции на $\Lambda_{0}^{2}$, обладающие свойствами $\operatorname{supp} \mathbf{e}_{j}(\psi) \in \Omega_{j}^{I}$, $\sum_{j} \mathbf{e}_{j}=1$. Нам также понадобится срезающая функция $\mathbf{e}_{0}(\alpha), \mathbf{e}_{0}(\alpha)=1$ при $|\alpha|<\delta / 2$, и $\mathbf{e}_{0}(\alpha)=0$ при $|\alpha|>\delta$, где $\delta>0$ достаточно мало.

2.7. Функция источника. Функция $V(y)$ порождает функцию на $\Lambda_{0}^{2}$ следующего вида:

$$
f(\rho, \psi)=\sqrt{\rho} \widetilde{V}(\rho \mathbf{n}(\psi)) .
$$

Произведение $f(\rho, \psi) \mathbf{e}_{0}(\alpha)$ определяет финитную функцию на $\Lambda_{0}^{2}$.

2.8. Частичное $h$-преобразование Фурье. Напомним, что в конструкции канонического оператора участвуют операторы преобразования Фурье по части переменных:

$$
\begin{aligned}
& {\left[\mathscr{F}_{p_{1} \rightarrow x_{1}}^{-h} \chi\left(p_{1}, x_{2}\right)\right]\left(x_{1}, x_{2}\right)=\frac{\sqrt{i}}{\sqrt{2 \pi h}} \int_{-\infty}^{\infty} \chi\left(p_{1}, x_{2}\right) e^{i p_{1} x_{1} / h} d p_{1},} \\
& {\left[\mathscr{F}_{p_{2} \rightarrow x_{2}}^{-h} \chi\left(x_{1}, p_{2}\right)\right]\left(x_{1}, x_{2}\right)=\frac{\sqrt{i}}{\sqrt{2 \pi h}} \int_{-\infty}^{\infty} \chi\left(x_{1}, p_{2}\right) e^{i p_{2} x_{2} / h} d p_{2}, \quad \sqrt{i}=e^{i \pi / 4}}
\end{aligned}
$$

3. Канонический оператор Маслова на $\Lambda_{0}^{2}$ и основная теорема. Согласно [1] канонический оператор определен следующей формулой:

$$
\begin{aligned}
& K_{\Lambda_{0}^{2}}^{h}[f] \equiv \sum_{j=1,3} \mathscr{F}_{p_{2} \rightarrow x_{2}}^{-h}\left\{\frac{\exp \left(i\left(\int\langle p, d x\rangle-x_{2} p_{2}\right)(\psi, \alpha) / h\right)}{\sqrt{\left|\partial\left(x_{1}, p_{2}\right) / \partial(\alpha, \psi)\right|}}\right. \\
& \left.\times\left. f(\rho, \psi) \mathbf{e}_{j}(\psi)\right|_{\psi=\psi_{j}\left(x_{1}, p_{2}\right), \alpha=\alpha_{j}\left(x_{1}, p_{2}\right)}\right\} \\
& +\sum_{j=2,4} \mathscr{F}_{p_{1} \rightarrow x_{1}}^{-h}\left\{\frac{\exp \left(i\left(\int\langle p, d x\rangle-x_{1} p_{1}\right)(\psi, \alpha) / h\right)}{\sqrt{\left|\partial\left(p_{1}, x_{2}\right) / \partial(\alpha, \psi)\right|}}\right. \\
& \left.\times\left. f(\rho, \psi) \mathbf{e}_{j}(\psi)\right|_{\psi=\psi_{j}\left(p_{1}, x_{2}\right), \alpha=\alpha\left(p_{1}, x_{2}\right)}\right\} \\
& \equiv \frac{\sqrt{i}}{\sqrt{2 \pi h C_{0}}}\left\{\sum_{j=1,3} \int_{-\infty}^{+\infty} \frac{\exp \left(i\left(C_{0} \alpha \cos ^{2} \psi+x_{2} p_{2}\right) / h\right)}{|\cos \psi|}\right. \\
& \times\left. f(\rho, \psi) \mathbf{e}_{j}(\psi)\right|_{\psi=\psi_{j}\left(x_{1}, p_{2}\right), \alpha=\alpha_{j}\left(x_{1}, p_{2}\right)} d p_{2} \\
& +\sum_{j=2,4} \int_{-\infty}^{+\infty} \frac{\exp \left(i\left(C_{0} \alpha \sin ^{2} \psi+x_{1} p_{1}\right) / h\right)}{|\sin \psi|} \\
& \left.\times\left. f(\rho, \psi) \mathbf{e}_{j}(\psi)\right|_{\psi=\psi_{j}\left(p_{1}, x_{2}\right), \alpha=\alpha_{j}\left(p_{1}, x_{2}\right)} d p_{1}\right\} .
\end{aligned}
$$


Сделаем в интегралах замену переменных по формулам $p_{2}=\sin \psi, p_{1}=\cos \psi$. С учетом знаков синуса и косинуса в соответствующих картах получим

$$
\begin{aligned}
K_{\Lambda_{0}^{2}}^{h}[f]= & \frac{\sqrt{i}}{\sqrt{2 \pi h C_{0}}}\left\{\left.\sum_{j=1,3} \int_{0}^{2 \pi} \exp \frac{i\left(C_{0} \alpha \cos ^{2} \psi+x_{2} \sin \psi\right)}{h} f(\rho, \psi) \mathbf{e}_{j}(\psi)\right|_{\alpha=\alpha_{j}\left(x_{1}, \sin \psi\right)} d \psi\right. \\
& \left.+\left.\sum_{j=2,4} \int_{0}^{2 \pi} \exp \frac{i\left(C_{0} \alpha \sin ^{2} \psi+x_{1} \cos \psi\right)}{h} f(\rho, \psi) \mathbf{e}_{j}(\psi)\right|_{\alpha=\alpha_{j}\left(\cos \psi, x_{2}\right)} d \psi\right\} \\
= & \frac{\sqrt{i}}{\sqrt{2 \pi h C_{0}}} \int_{0}^{2 \pi} \exp \frac{i\left(x_{1} \cos \psi+x_{2} \sin \psi\right)}{h} f(\rho, \psi) \sum_{j=1}^{4} \mathbf{e}_{j}(\psi) d \psi \\
= & \frac{\sqrt{i}}{\sqrt{2 \pi h C_{0}}} \int_{0}^{2 \pi} \exp \frac{i\left(x_{1} \cos \psi+x_{2} \sin \psi\right)}{h} f(\rho, \psi) d \psi .
\end{aligned}
$$

Полагая $h=\mu / \rho$ и используя (6), получаем

$$
\begin{aligned}
K_{\Lambda_{0}^{2}}^{\rho / \mu}[f] & =\frac{\sqrt{i} \rho}{\sqrt{2 \pi \mu C_{0}}} \int_{0}^{2 \pi} \exp \frac{i \rho\langle x, \mathbf{n}(\psi)\rangle}{\mu} \widetilde{V}(\rho \mathbf{n}(\psi)) d \psi \\
\int_{0}^{\infty} K_{\Lambda_{0}^{2}}^{\rho / \mu}[f] d \rho & =\frac{\sqrt{i}}{\sqrt{2 \pi \mu C_{0}}} \int_{0}^{\infty} \int_{0}^{2 \pi} \exp \frac{i \rho\langle x, \mathbf{n}(\psi)\rangle}{\mu} \widetilde{V}(\rho \mathbf{n}(\psi)) d \psi d \rho=\frac{\sqrt{2 \pi i}}{\sqrt{\mu C_{0}}} V\left(\frac{x}{\mu}\right) .
\end{aligned}
$$

Таким образом, доказана

Теорема 1. Пусть $\Lambda_{0}^{2}$ - лагранжево многообразие (5) и $f(\rho, \psi)$ определено в (6). Тогда справедливо равенство (4).

4. Локализация функции на $\Lambda_{0}^{2}$. Функция $V(x / \mu)$ локализована вблизи начала координат. Используя это обстоятельство, можно ограничиться рассмотрением не всего многообразия $\Lambda_{0}^{2}$, а только окрестности кривой $\Gamma_{0}=\left\{p=\mathbf{n}(\psi), x=0, \psi \in \mathbb{S}^{1}\right\}$. Другими словами, функцию $f(\rho, \psi)$ можно заменить на финитную функцию $f(\rho, \psi) \mathbf{e}(|\alpha|)$ на $\Lambda_{0}^{2}$, где

$$
\mathbf{e}(z)=\left\{\begin{array}{cc}
1, & 0 \leqslant z \leqslant \delta / 2 \\
0, & z \geqslant \delta
\end{array}\right.
$$

Теорема 2. Пусть $V(y)$ убывает быстрее любой степени $|y|^{-k}$ при $|y| \rightarrow \infty, k \geqslant 2$. Тогда

$$
V=\left(\frac{x}{\mu}\right)=\left.\sqrt{\frac{\mu C_{0}}{2 \pi i}} \int_{0}^{\infty} K_{\Lambda_{0}^{2}}^{h}[f(\rho, \psi) \mathbf{e}(|\alpha|)]\right|_{h=\mu / \rho} d \rho+O\left(\mu^{2}\right) .
$$

ДоказАтельство. Представим $f$ в виде $f=f \mathbf{e}+f(1-\mathbf{e})$ и оценим вклад от второго слагаемого в формулу (4). Заметим, что носитель функции $1-e$ не пересекается с циклом особенностей $\Lambda_{0}^{2}$, поэтому результат применения канонического оператора к этой функции $\bmod O(\mu)$ совпадает с функцией

$$
\sqrt{\frac{\mu C_{0}}{2 \pi i}} \frac{1}{|x|} \int_{0}^{\infty}\left(e^{i \rho|x| / \mu} \widetilde{V}\left(\rho \frac{x}{|x|}\right)+e^{i \rho|x| / \mu} \widetilde{V}\left(-\rho \frac{x}{|x|}\right)\right) \sqrt{\rho}(1-\mathbf{e}(|\alpha|)) d \rho .
$$

Теперь утверждение теоремы вытекает из очевидной оценки

$$
\int_{0}^{\infty} e^{i \rho / \mu} u(\rho) \sqrt{\rho} d \rho=\frac{\mu}{i} \int_{0}^{\infty} u(\rho) \sqrt{\rho} d\left(e^{i \rho / \mu}\right)=-\frac{\mu}{i} \int_{0}^{\infty} e^{i \rho / \mu} \frac{\partial}{\partial \rho}(u(\rho) \sqrt{\rho}) d \rho=O(\mu),
$$

справедливой для любой быстро убывающей функции $u(\rho)$. 


\section{СПИСОК ЦИТИРОВАННОЙ ЛИТЕРАТУРЫ}

[1] В.П. Маслов, М.В. Федорюк, Квазиклассическое приближение для уравнений квантовой механики, Наука, М., 1976. [2] В.П. Маслов, М.В. Федорюк, Матем. заметки, 45:5 (1989), 50-62. [3] С. Ю. Доброхотов, П.Н. Жевандров, В.П. Маслов, А.И. Шафаревич, Матем. заметки, 49:4 (1991), 31-46. [4] С. Ю. Доброхотов, С. Я. Секерж-Зенькович, Б. Тироцци, Т. Я. Тудоровский, Докл. РАН, 409:2 (2006), 171-175. [5] F. Treves, Introduction to Pseudodifferential and Fourier Integral Operators, vol. 1: Pseudodifferential operators. vol. 2: Fourier integral operators, The University Series in Mathematics, Plenum Press, New York-London, 1980.

С. Ю. Доброхотов

Институт проблем механики РАН

12.06.2005

E-mail: dobr@ipmnet.ru

\section{Б. Тироцци}

Университет "La Sapienza", Рим

E-mail: b.tirozzi@libero.it

\section{А. И. Шафаревич}

Институт проблем механики РАН

E-mail: shafar@mech.math.msu.su 